\title{
RÉPERTOIRE
}

\section{D'ESPÈCES ET DE GENRES NOUVEAUX}

\section{Rhizopodes}

Endamoeba Iutea Henderson (J. C.). Amoebidae. Cubitermes (Isoptère). Univ. California. Publ. Zool., XLIII, 1941 (14).

Entamoeba pellucida Henderson (J. C.). Endamoebidae. Cubilermes (Isoptère). Univ. California. Publ. Zool., XLIII, 1941 (14).

Entamoeba pyrrhogaster E. A. Lobeck. Endamoebidæ. Intestin. Trilurus pyrrhogaster (Batr.). Japon. Jl. of Paras., XXVI, 1940, p. 243.

Entamoeba Salamandr.ze E. A. Lobeck. Endamoebidæ. Intestin. Triturus pyrrhogasler (Batr.), Japon. Jl. of Paras., XXVI, 1940, p. 243.

Entamoeba ctenosaurae R. Hegner et R. Hewitt. Endamoebida. Intestin. Iguane: Clenosaura acanthura (Rept.). Mexique. Jl. of Paras., XXVI, 1940 , p. 319.

Entamoeba cuautlae R. Hegner et R. Hewitt. Endamoebidæ. Intestin, Ctenosaura acanthura (Rept.). Mexique. Jl. of Paras., XXVI, 1940, p. 319.

Entamoeba dobelli F. da Fonseca. Endamoebida. Intestin. Tapilus raulinus (Mamm.) Brésil. Mem. Inst. Bulantan, São Paulo, XIV, 1940, p. 43.

Endolimax goheeni Mello et Lobo. Amoebida. Intestin. Coptermes (Isoptère). Inde. Proc. Indian. Acad. Sci., XIV B, 1941, p. 75.

Endolimax suggrandis J. C. Henderson. Amoebidæ. Cubitermes (Isoptère). Univ. California. Publ. Zool., XLIII, 1941 (14) p. 13.

Copramoeba E. A. Lobeck. Amoebidæ. Espèce type : C. salamandræ E. A. Lobeck. $J l$. of Paras., XXVI, 1940, p. 243.

Copramoeba salamandrae E. A. Lobeck. Amoebida. Intestin. Trilurus viridescens (Batr.). États-Unis. Jl. of Paras., XXVI, 1940, p. 243.

Dobellina A. Bishop et P. Tate. Amoebidx. Espèce type: D. mesnili (= Entamoeba mesnili Keilin 1917). Parasilology, Cambridge, XXXI, 1940 , p. 501.

Dobellina rayi Misra. P. L. Amoebidæ. Varanus monitor. Indes. Proc. Indian Sci. Congr., XVIII (3), p. 169.

Martineziella baezi R. Hegner et R. Hewitt. Amoebidæ. Ctenosaura acanthara (Rept). Mexique. Jl. of Paras., XXVI, 1940, p. 319 et XXVII, 1941, p. 42.

Vahlkampfia salamandrae E. A. Lobeck. Amoebidæ. Intestin. Trilurus pyrrhogasler (Batr.). Japon. Jl. of Paras., XXVI, 1940, p. 243.

M. ANSEL.

Ann. de Parasitologie, T. XXII, $\mathrm{N}^{\circ \mathrm{s}} 5-6,1947$, p. $482-491$. 


\section{Flagellés}

Leptomonas anophelini da Fonseca J. A. B. et da Fonseca F. Trypanosomidr. Anopheles eiseni (Dipt.). Brésil. Mem. Inst. Bulanlan, São Paulo, XV, 19t2, p. 101.

Crithidia gallardoi Mazza. Trypanosomidæ. Intestin. Microlomus lucifer (Berg), Reduviida (Hétéroptère). Publ. Mission Estud. Patol. Reg. Argent, Jujuy, XXXII, 1937, p. 37.

C.Ithidia penicillidiae Reichenow E. Trypanosomidr. Intestin. Penicillidia pachymella, Nycleribiidæe (Dipt.). Afrique orient. Archiv für Protislenkunde, XCIV, 1940. p. 267.

Herpetomonas leptocoridis Mc. Culloch. Nom. nov. pro Crilhidia leplocoridis Mc. Culloch. Trypanosomida. Tube digestif. Leplocoris trivillatus (Hémipt.). Trans. Amer. Micr. Soc., 1942, p. 120.

Herpetomonas pessoai Ayrosa Galvâo A. L. et Coutinho J. O. Trypanosomidær. Intestin. Anopheles sp. Culicida (Dipt.). Brésil. Rev. Bras. Biol., I, 1941, p. 311.

Trypanosoma fulicae da Fonseca L. Trypanosomida. Fulica alra. Rallidæ (Ois.). Indes. Arq. Esc. Medic. cirurg. Nova-Goa, XII, 1938, p. 315 .

Trypanosoma gaumontis Fantham H. B., Porter A. et Richardson L. R. Trypanosomidar. Bufo Americanus (Batr.). Canada oriental. Parasitology, XXXIV, 1942, p. 199.

Trypanosoma knowlesi Das Gupta B. M. et Siddons L. B. Trypanosomidz. Uroloncha malabarica (Ois). Indes. Ind. Med. Gaz., LXVI, 1941, p. 151 .

Trypanosoma Iavalia Fanthan H. B., Porter A. et Richardson L. R. Trypanosomidx. Bufo americanus (Batr.). Est du Canada. Parasilolog!, XXXIV, 1912, p. 199.

Trypanosoma mpspuensis Reichenow E. Trypanosomidr. Nycleris athiopica (Mamm.). Afrique orientale. dreh. für l'rolistenkunde, XCIV, 1940 , p. $267-287$.

Trypanosoma montrealis Fantham H. B., Porter, A. et Richardson L. R. Trypanosomidæ. Bufo americanus. Canada oriental. Parasilology, XXXIV, 1942 , p. 199.

Trypanosoma myoxocephali Fantham H. B., Porter A. et Richardson L. R. Trypansomidx. Myoxocephalus oclodecimspinosus. Parasilology, XXXIV, 1942, p. 199.

Trypanosoma cnae Sergent Ed. Trypanosomida. Oena capensis. Columbidæ (O s.). Sahara. Arch. Inst. Past. Alger, XIX, 1941, p. 431.

Trypanosoma sigmodoni Culbertson J. T. Trypanosomidx. Sigmodon hispidus "Cotton rat» (Mamm.). Floride. Jl. of Paras., XXVII, 1941, p. 45.

Trypanosoma uroloxchae Pereira J. Trypanosomida. Uroloncha slriata. (Ois.). Indes. Proc. Ind. Acad. Sci., XIII, 1941, B, p. 33.

Costia pyriformis Davis H. S. Tetramilidæ. Salmo iridens el Salvelinus fontinalis (Poiss.). Etats-Unis. Jl. of Paras., XII, 1943, p. 385. 
Chilomastix navasi F. da Fonseca. Chilomastigida. Intestin. Tapirus sp. Brésil. Mem. Insl. Butantan São Paulo, XIV, 1940, p. 43.

Eutrichomastix bertholdoi F. da Fonseca. Polymastigidæ. Intestin. Tapirus sp. Brésil. Mem. Inst. Bulantan, São Paulo, XIV, 1940, p. 43.

Pentatrichomastix eutamias Tanabe M. et Okinami M. Polymastigida. Eulamias asialicus (Mamm.). Japon. Keizyo. J. Med., X, 1940, p. 126.

Barroella Zeliff. nom. nov. pro. Kirbyella Zeliff 1930 (= Proboscidiella Kofoïd et Swezy). Jl. of Paras., XXX, 1944, p. 275.

Devescovina arta Kirby H. Devescovinidre. Intestin. Glyptotermes angustus (Isoptère). Panama. Univ. Calif. Publ. Zool., XLV, 1942, p. 93.

Devescovina coghilli Kirby H. Devescovinida. Cryplotermes merwei (Isoptère). Afrique du Sud. Univ. Calif. Publ. Zool., XLV, 1942, p. 93.

Devescovina endicula Kirby $\mathrm{H}$. Devescovinida. Kalolermes jeannelanus (Isoptère). Afrique orientale. Univ. Calif. Publ. Zool., XLV, 1942, p. 93.

Devescovina exilis Kirby H. Devescovinida. Neolermes connexus (Isoptère). Hawaī. Univ. Calif. Publ. Zool., XLV, 1942, p. 93.

Devescovina fissa Kirby H. Devescovinida. Procryplolermes. sp. (Isoptère). La Réunion. Univ. Calif. Publ. Zool., XLV, 1942, p. 93.

Devescovina hawaïensis Kirby H. Devescovinidæ. Nom. nov. pro. Devescovina striala. var. hawaïensis. Univ. Calif. Publ. Zool., XLV, 1942, p. 93.

Devescovina insolita Kirby H. Devescovinida. Neotermes, sp. (Isoptère). Tanganyika. Univ. Calf. Publ. Zool., XLV, 1942, p. 93.

Devescrvina lepida Kirby H. Devescovinida. Calcaritermes brevicollis (Isoptère). Panama. Univ. Calif. Publ. Zool., XLV, 1942, p. 93.

Devescovina parasoma Kirby H. Devescovinida. Neotermes teclonæ (Isoptère). Indes néerlandaises. Univ. Calif. Publ. Zool., XLV, 1942, p. 93.

Devescovina robusta Kirby H. Devescovinida. Neolermes erylhraeus (Isoptère). Erythrée. Univ. Calif. Publ. Zool., XLV, 1942, p. 93.

Davescovina similis Kirby H. Devescovinida. Cryplotermes sp. (Isoptère). Madagascar. Univ. Calif. Publ. Zool., XLV, 1942, p. 93.

Devescovina transita Kirby H. Devescovinidre. Glyptolermes. sp. (Isoptère). Java. Univ. Calif. Publ. Zool., XLV, 1942, p. 93.

Devescovina uniflexura Kirby H. Devescovinida. Kalolermes perezi (Isoptère). Costa-Rica. Univ. Calif. Publ. Zool., XLV, 1942, p. 93.

Devescovina vestita Kirby H. Devescovinida. Glyplotermes niger (Isoptère). Java. Univ. Calif. Publ. Zool., XLV, 1942, p. 93.

Devescovina vittata Kirby H. Devescovinidæ. Kalolermes. sp. (Isoptère). Tanganyika. Univ. Calif. Publ. Zool., XLV, 1942, p. 93.

Caduceia bugnioni Kirby H. Devescovinidæ. Neotermes greeni (Isoptère). Ceylan. Univ. Calif. Publ. Zool., XLV, 194?, p. 93.

Caduceia kalshoveni Kirby H. Devescovinida. Neolcrmes dalbcrşiae (Isoptère). Java. Univ. Calif. Publ. Zool., XLV, 1942, p. 93.

Caduceia monile Kirby H. Devescovinidæ. Neolermes teclonæ (Isoptère) Indes Néerlandaises. Univ. Calif. Pabl. Zool., XLV, 1942, p. 93. 
Macrotrichomonas lighti Kirby H. Nom. nov. pro. Gigantomonas lighti. Devescovinida. Univ. Calif. Publ. Zool., XLV, 1942, p. 93.

Macrutrichomonas procera Kirby H. Devescovinidæ. Calcarilermes brevicollis (Isoptère). Panama. Univ. Calif. Publ. Zool., XLV, 1942, p. 93.

Macrotrichomonas ramosa Kirby H. Devescovinida. Glyptolermes brevicaudatus (Isoptère). Java. Univ. Calif. Publ. Zool., XLV, 1942, p. 93.

Macrotrichomonas restis Kirby H. Devescovinidx. Kalotermes jouleli (Isoptère). Floride. Univ. Calit. Publ. Zool., XLV, 1942, p. 93.

Macrotrichomonas unguis Kirby H. Devescovinida. Glyptolermes caudomunitis (Isoptère). Java. Univ. Calif. Publ. Zool., XLV, 1942, p. 93.

Macrotrichomonss virgosa Kirby $H$. Devescovinida. Procryptolermes sp. (Isoptère). Madagascar. Univ. Calif. Publ. Zool., XLV, 1942, p. 93.

Trichomonas hoplodactyli Percival E. Trichomonadida. Hoplodactylus maculatus (Rept.). Nel.e-Zélande. Rec. Canterbury (N Z) Mus., IV, 1941, p. 373.

Trichomonas tapiri da Fonseca F. Trichomonadidx. Tapirus (Mamm.). Brésil. Mem. Inst. Bulanlan, São Paulo, XIV, 1940, p. 43.

Giardia beltrani Larenas M. R. Hexamitidx. Intestin. Passer domesticus (Ois.). Rev. Soc. Mexic. Hist. Nat., V, 1944, p. 43.

Hexamita eufamias Tanabe M. et Okinami M. Hexamilidx. Eulamias asialicus. Sciuridæ (Mamm.). Japon. Keizyo. J. Med., X, 1940, p. 126-134.

Hexamita meleagridis Mc. Neil E, Hinshaw W. R. et Kofoïd C. A. Hexamitidx. Dindon (Ois.). Amer. Journ. Hygiene, XXXIV, 1941, p. 71.

Calonympha avita Brown. H. E. Calonymphida. Cryptelermes. sp. (Isoptère). États-Unis. J. Tenn. Acad. Sci., XVI, 1941, p. 357.

Calonympha grandis Brown. H. E. Calonymphidie. Rugilermes. sp. (Isoptère). États-Unis. J. Tenn. Acad. Sci., XVI, 1941, p. 357.

Calonympha patella Brown. H. E. Calongmphidæ. Neolermes. sp. (Isoptère). J. Tenn. Acad. Sci., XVI, 1941, p. 357.

Coronympha Kirby H. Calonymphida. Espèce type : C. octonaria Kirby H. Proc. Calif. Acad. sc. (4). XXII, 1939, p. 207.

Coronympha octonaria Kirby H. Calonymphidæ. Kalotermes emersoni (Isoptère). Mexique. Proc. Calif. Acad. Sci., (4). XXII, 1939, p. 207.

Metacoronympha Kirby H. Calonymphidx. Espèce type : M. senta Kirby H. Proc. Calif. Acad. Sc., (4). XXII, 1939, p. 207.

Metacoronympha senta Kirby H. Calonymphidæ. Intestin. Kalolermes emersoni (Isoptère). Mexique. Proc. Calif. Acad. Sci., (4). XXII, 1939, p. 207.

Holomastigotö̈des nilotica Jennings C. Holomastigolidx. Prorhenotermes (Isoptère). États-Unis. J. Tenn. Acad. Sci., XVII, 1942, p. 343.

Trichonympha ampla Kirby H. Trichonymphidæ. Intestin. Kalotermitidæ (Isoptères). Univ. Calif. Publ. Zool., XLIX, 1944, p. 185.

Trichonympha corbula Kirby H. Trichonymphida. Intestin. Kalolermitides (Isoptères). Univ. Calif. Publ. Zool., XLIX, 1944, p. 185.

Trichonympha divexa Kirby H. Trichonymphida. Intestin. Kalolermilides (Isoptères). Univ. Calif. Publ, Zool., XLIX, 1944, p. 185. 
Trichonympha peplophora Kirby H. Trichonymphidæ. Intestin. Kalotermilides (Isoptères). Univ. Calif. Publ. Zool., XLI , 1944, p. 185.

Trichonympha teres Kirby H. Trichonymphida. Intestin. Kalolermitides (Isoptères). Univ. Calif. Publ. Zool., XLIX, 1914, p. 185.

Polyflagellomonas Carpano M. Espèce type : P. genilalis Carpano. M. Riv. di Parassil., Roma. IV, 1940, p. 133.

Polyfiagellomonas geniralis Carpano M. Exsudat vaginal. Equus caballus. Italie. Rivisla di Parasilologia IV, 1910, p. 133.

M. ANsEr.

\section{Nématodes}

Austrostrongylus aggregatus Johnston et Mawson. Strongylidæ. Duodénum. Macropus ualabatus (Marsup.). Australie. Proc. Linn. Soc. N. S.W., LXV, 1940, p. 472.

Brevistriata sinensis Li. Trichoslrongylidx. Intestin, cacum. Callosciurus erythræus caloneoientralis (Mamm.). Chine. Peking Nal. His'. Bull., XV, 1941, 195.

Citellinema nipponicum Yamaguti. Trichostrongylidre. Intestin. Sciurus lis (Mamm.). Japon. Japan. J. Zool., IX, 1941, p, 418.

Cloacina digitata Johnston et Mawson. Trichonemalidæ. Macropus dorsalis (Marsup.). Australie. Proc. Linn. Soc. N.S.W., LXV, 1940, p. 469.

Cloacina linstowi Johnston et Mawson. Trichonematidæ. Macropus ruficollis (Marsup.). Australie. Proc. Linn. Soc. N.S.W., LXV, 1940, p. 469.

Cloacina gallardi Johnston et Mawson. Trichonemalidæ. Macropus ualabatus (Marsup.). Proc. Linn. Soc. N.S.W., LXV, 1940, p. 470.

Contracaecum osculatum Lyster. Heterocheilidæ. Phoques. Arctique. Canad. J. Res., Ollawa, XVIII, D, 1940, p. 398.

Contracaecum macquariae Johnston et Mawson. Heterocheilidx. Estomac. Macquaria australasica (Perche) (Poiss.). Australie. Trans. Roy. Soc. S. Austr., LXIV, 1940, p. 343.

Contracaecum epinepheli Yamaguti. Helerocheilidæ. Intestin grèle, Epinephelus akaara (Poiss.). Japon. Japan. J. Zool., IX, 1941, p. 358.

Contracaecum seriolae Yamaguti. Heterocheilida. Estomac. Seriola quinquera tiala (Poiss.). Japon. Japan. J. Zool., IX, 1941, p. 359.

Contracaecum paralichthycis Yamaguti. Helerocheilidæ. Estomac. Paralichthydis olivaceus (Poiss.). Japon. Japan. J. Zool., IX, 1941, p. 360.

Contracaecum scomberomori Yamaguti. Heterocheilidæ. Scomberomorus chinensis (Poiss.). Japon. Japan. J. Zool., IX, 1941, p. 362.

Contracaecum coiliae Yamaguti. Helerocheilidæ. Estomac. Coilia mystus (Poiss.). Japon. Japan. J. Zool., IX, 1941, p. 363.

Contracaecum baylisi Yamaguti. Helerochellidae, Zeus japonicus (Poiss.). Japon. Japan. J. Zool., IX, 1941, p. 264.

Contracaecum ilishae Yamaguti. Hcterocheilidæ. Estomac. Ilisha elongata (Poiss.). Japon. Japan. J. Zool., IX, 1941, p. 365. 
Contracaecum zenopsis Yamaguti. Heterocheilidæ, Zenopsis nebulosa (Poiss.). Japon. Japan. J. Zool., IX, 1941, p. 366.

Contracaecum saba Yamaguti. Heterocheilidæ. Scomber japonicus (Poiss.). Japon. Japan. J. Zool., IX, 1941, p. 367.

Contracaecum nycticoracis Johnston et Mawson. Heterocheilidx. Nyclicorax caledonicus (Ois.). Australie. Rec. S. Austr. Mus. Sydney, XXI, 1941 , p. 15.

Contracaecum quincuspis Lucker. Helerocheilidx. Anhinga anhinga (Ois.). U.S.A. J. Wash., Acad. Sc., XXXI, 1941, p. 33.

Contracaecum himeu Yamaguti. Heterocheilidx. Oiseaux. Japon. Japan. J. Zool, IX, 1941, p. 457.

Contracaecum umiu Yamaguti. Helerocheilidæ. Oiseaux. Japon. Japan. J. Zool., IX, 1941, p. 459.

Contracaecum bancrofti Johnston et Mawson. Helerocheilidæ. Pelecanus conspicillalus (Ois.). Australie. Trans. Roy. Soc. S. Austr., LXV, 1941. p. 112.

Contracaecum clelandi Johnston et Mawson. Heterocheilidæ. Pelecanus conspicillalus (Ois.). Australie. Trans. Roy. Soc. S. Auslr., LXV, 1941, p. 113.

Contracaecum sinulabiatum Johnston et Mawson. Helerocheilidæ. Plolus novæ hollandiuæ (Ois.). Australie. Trans. Roy. Soc. S. Auslr., LVX, 1941, p. 113.

Contracaecum magnicollare Johnston et Mawson. Heterocheilidx. Anous stolidus (Ois.). Australie. 1rans. Roy. Soc. S. Austra., LXV, 1941, p, 114.

Crenosoma microbiusa Wallace. Metastrongylidx. Bronches. Mephilis minnesole (Mamm.). Minnesota. Proc. Helminlh. Soc. Washington, VIII, 1941, p. 58 .

Crenosoma mephitidis Hobmaier. Melastrongylida. Bronches. Spilogale gracilis, Mephilis occidentalis (Mamm.). Californie. J. Parasit. Urbana, XXVII, 1941, p. 229.

Cyclostrongylus medioannulatus Johnston et Mawson. Trichoneminæ. Macropus thetis (Marsup.). Australie. Proc. Linn. Soc. N.S.W., LXV, 1941 , p. 471.

Gazia fluviatills Johnston et Mawson. Helerocheilidæ. Ouïes. Pleclroplites ambiguus, MacCullcchella macquariensis, Percalales colonorum (Poiss.). Australie. Trans. Roy. Soc. S. Austr., LXIV, 1940, p. 342.

Heterakis yamadori Yamaguti. Heterakidæ. Cæcum. Syrmalicus sœmerringi (Ois.). Japon. Japan. J. Zool., IX, 1941, p. 447.

Labiostomum Akhtar. Oxyuridæ. Espèce type: L. naimi Akhtar. Rec. Ind. Mus. Calcuta, XLIII, 1941, p. 217.

Labiostomum naimi Akhtar. Oxyuridre. Intestin. Ochotona sp. (Mamm). Afghanistan. Rec. Ind. Mus. Calcutta, XLIII, 1941, p. 217.

Longistriata musasabi Yamaguti. Trichostrongylidæ. Intestin grêle. Petaurista leucogenys nikkonis (Mamm.). Japon. Japan. J. Zool., IX, 1941, p. 413.

Megalobatrachonema Yamaguti. Kathlaniidæ. Espèce type : $M$. nipponicum Yamaguti. Japan. J. Zool., IX, 1941, p. 403. 
Megalobatrachonema nipponicum Yamaguti. Kalhlaniidæ. Intestin. Megalobalrachus juponicus (Amph.). Japon. Japan. J. Zool., IX, 1941, p. 403.

Moguranema Yamaguti. Trichostrongylidx. Fspèce type: $M$. nipponicum Yamaguti. Japan. J. Zool., IX, 1941, p. 416.

Moguranema nipponicum Yamaguti. irichostrongylidre. Intestin grêle. Mogera wogura (Mamm.). Japon. Japan. J. Zool., 1X, 1941, p. 412.

Molinostrongyl's rhinolophi Yamaguti. Trichostrongylida. Intestin. Rhinolophus ferrum-equinum (Mamm.). Japon. Japan. J. Zool., IX, 1941 , p. 412.

Oswaldocruzia peipingensis Chow. Trichoslrongylida. Intestin grêle. Bufo bufo (Batr.). Chine. Chin. Med. J. Suppl., III, 1940, p. 292.

Paranisakiopsis Yamaguti. Anisakinæ. Espèce type : P. cœlorhynchi Yamaguti, Japan. J. Zool., IX, 1941, p. 350.

Paranisakiopsis cœlorhynchi Yamaguti. Anisakinæ. Intestin. Colorhynchus sp. (Poiss.). Japon. Japan. J. Zool., IX, 1941, p. 350.

Paranisakis halieutaeae Yamaguti. Anisakinæ. Intestin gı êle. Halieutaea slellata (Poiss.). Japon. Japan. J. Zool., IX, 1941, p. 349.

Pharyngodon polypedatis Yamaguti. Oxyuridre, Intestin. Polypedales buergeri (Amph.). Japon. Japan. J. Zool., 1X, 1941, p. 401.

Phocascaris netsiki Lyster. Ascaridx. Phoques. Canada. Canad. J. Res. Ollawa, XVIII, D, 1940 , p. 399.

Porrocaecum clelandi Johnston et Mawson. Anisakinæ. Oreocincla lunulata, Cinclosoma punctalum, Anthus australis (Ois.). Australie et Tasmanie. Proc. Linn. Soc. N.S. W., LXVI, 1941, p. 252.

Porrocaecum streperae Johnston et Mawson. Anisakina. Strepera versicolor (Ois.). Australie. Proc. Linn. Sic. N.S. W., LXVI, 1941, p. 253.

Porrocaecum circinum Johnston et Mawson. Anisakinæ. Circus assimilis, Nisælus morphnoides, Astur nova-hollandiæ (Ois.). Australie. Trans. Roy. Soc. S. Auslr., LXV, 1941, p. 30.

Porrocaecum cephaloscyllii Yamaguti. Anisakinæ. Estomac. Cephaloscyllium umbratile (Poiss.). Japon. Japan. J. Zool., IX, 1941, p. 357.

Porrocaecum phalacrocoracis Yamaguti. Anisakinr. Oiseaux. Japon. Japan. J. Zool., IX, 1941, p. 454.

Pseudanisakis Yamaguti. Helerocheilida. Espèce type: P. rajæ Yamaguti. Japan. J. Zool. IX, 1941, p. 347.

Pseudanisakis rajae Yamaguti. Heterocheilidx. Estomac. Raja kenojei (Poiss.). Japon. Japan. J. Zool., IX, 1941, p. 347.

Raphidascaroides Yamaguti. Heterocheilidæ. Espèce type: R. nipponensis Yamaguti. Japan. J Zool., IX, 1941, p. 353.

Raphidascaroides nipponensis Yamaguti. Helerocheilidæ. Intestin. Ateleops's japonicus, Zebrias zebrinus (Poiss.). Japon. Japan. J. Zool. IX, 1941, p. 353.

Rhabdias polypedatis Yamaguti. Rhabdiasidæ, Poumon. Polypedales buergeri (Amphib.). Japon. Japan. J. Zool., IX, 1941, p, 397.

Rhabdias rhacophori Yamaguti. Rhabdiasida. Rhacophorus schlegell (Amph.). Japon. Japan. J. Zool., IX, 1941, p. 398. 
Spinostrongylus sinensis Wu et Liu. Trichoslrongylida. Intestin. Chauvesouris. Kwangsi, Chine. Sinensia, Nanking, XI, 1940, p. 398.

Spironoura elsøyae Johnston et Mawson. Kalhlaniida. Elseya denlala (Rept.). Australie. Rec. Austr. Mus. Sydney, XXI, 1941, p. 15.

Syironoura rankini Walton. Kalhlaniida. Intestin. Rana clamilans (Batr.). Maine. J. Parasil. Urbana, XXVII, 1941, p. 180.

Strongyluris tumefaciens Price et Dikmans. Helerakinæ. Tumeurs de l'intestin. Chats. U.S.A. Proc. Helminth. Soc. Washinglon, VIII, 1941, p. 41.

Strongyluris acaudata Caballero. Helerakina. Intestin. Sceloporus ferrariperezi (Rept.). La Havane. Rev. Med. Trop. Habana, VII, 1941, p. 31.

Subulura peragale Johnston et Mawson. Subulurida. Estomac. Peragale minator (Marsup.). Australie. Proc. Linn. Soc. N.S. W., LXV, 1940, p. 472.

Subulura clelandi Johnston et Mawson. Subulurida. Podargus strigoides (Ois.). Australie. Proc. Linn. Soc. N.S. W., LXVI, 1941, p. 251.

Subulura minetti Bhalerao. Subulurida. Oiseaux de basse-cour. Indes. Proc. Ind. Acad. Sc. IV, B, 1941, p. 339.

Subulura coturnicis Yamaguti. Subuluridæ. Colurnix colurnix(Ois.). Japon. Japan. J. Zool., IX, 1941, p. 449.

Toxocara tanuki Yamaguti. Ascarida. Intestin grêle. Nycterentes procyonoides (Ois.). Japon. Jafan. J. Zool., 1X, 1941, p. 421.

Acuaria corvicola Johnston et Mawson. Acuariida. Estomac. Corbeau. Australie. Rec. Austr. Mus. Sydney, XXI, 1941, p. 10.

Acusria flindersi Johnston et Mawson. Acuariidx. Hieracidea orienlalis (Ois.). He Flinders, Australie. Trans. Roy. Soc. S. Austr., LXV, 1941, p. 31.

Cheilospirura streperina Johnston \& Mawson. Actariida. Strepera fuliginosa (Ois.). Australie. Proc. Linn. Soc. N.S. W., LXVI, 1941, p. 254.

Anguillicola axstraliensis Johnston \& Mawson, Filarioidea. Vessie natatoire. Anguilla reinhardlii (Poiss.). Australie. Trans. Roy. Soc. S. Austr., LXIV, 1940 , p. 351 .

I. CAMPANA.

\section{Anoploures}

Prolinognathus arcuatus H. Fahrenholz. Procavia shoana? (Mamm.). Jardin zool. de Copenhague. Zlschr. f. Parasilenk., XI, 1939, p. 5.

Prolinognathus foleyi H. Fahrenholz. Procavia ru'escens bounhioli Kollm. (Mamm.). Alger. Zlschr. f. Parasilenk., XI, 1939, p. 9.

Prolinognathus ferrisi H. Fahrenholz. Procavia brucei rudolfi (Mamm.). Afrique orientale anglaise. Ztschr.f. Parasilenk., XI, 1939, p. 12.

Pro inognathus aethiopicus H. Fahrenholz. Procavia shoana? (Mamm.). Jardin zoologique de Copenhague. Zlschr.f. Parasilenk., XI, 193 . p. 13.

M. LANGERON.

Acariens

Gigan:o:aelaps F. da Fonseca. Laelaptida. Espèce type : G. vilzlhumi F. da Fonseca. Mem. Inst. Butanlan, XII, 1938-1939, p. 12.

Gigantolaelaps vitzthumi F, da Fonseca, Laelaptidær. Rats sauvages. Brésil, 
Limites des Etats de Minos Geræs et de Goias: Mem. Inst. Butanlan, XII, 1938-1939, p. 28.

Gigantslaelaps oudemansi F. da Fonseca. Laelaplidx. Sur e rat sylvestı e ». Brésil, Etat de Goias, Anapolis. Me n. Inst. Butantan, XII, 1938-1939, p. 15.

Giganto'aelaps gilmorei F. da Fonseca. Laelaptidæ. Sur « rat sylvestre ». Brésil, Etat de Goias, Anapolis. Mem. Inst. Bulanlan, XII, 1958-1939, p. 22.

Gigantolaelaps goyanensis F. da Fonseca. Laelaplidæ. Estrimys sp., Melachirops opossum, Anapolis, Etat de (ioias; Nectomys squamipes, Manguinhos, Rio de Janeiro, Brésil (Mamm.). Mem. Inst. Bulantan, XII, 1938-1939, p. 32.

Gigantolaelaps comatus F. da Fonseca, Laelaptidx. Sur « rat sylvestre». Brésil. Etat de St-Paul, Institut de Butantan. Mem. Inst. Butantan, XII, 1938-1939, p. 39.

Pneumonyssus caninum Chandler et Ruhe. Dermanyssidx. Sinus frontal. Chien. U.S.A. Michigan. State College, East Lansing. Jl. of paras., XXVI, 1940 , p. 39.

Apolonia S. Torres et W. Braga. Trombidida. Espèce type : A. tigipioensis S. Torres et W. Braga. Bol. Soc. brasileira med. veler., IX, 1939, p. 28.

Apolonia tigipioensis S. Torres et W. Bragar. Trombididæ. Gallus gallus domesticus (Ois.). Brésil, Tigipio, environs de Recife. Bol. Soc. brasileira med. veler., IX, 1939, p. 30.

Otocentor R. A. Cooley. Ixodidx. Espèce type : O. nilens (Neum.) (=Dermacentor nitens Neum.). Bull Nat. Inst. Hlth., Washington, no 171, 1938.

M. LANGeron.

Mallophages

Craspedonirmus S. Kéler. Nirmidæ. Espèce type: C. frontalus (Nitzsch). (= Nirmus fronlalus Nitzsch). Zlsch.f. Parasitenk., XI, 1939, p. 49.

Acronirmus S. Kéler. Nirmida. Espèce type : A. gracilis (Nitzsch). Ztsch.

f. Parasilenk., XI, 1939, p. 50.

Neodocophorus S. Kéler. Docophoridæ. Espèce type : N. cursor (Nitzsch). Zlsch.f. Parasilenk., XI, 1939, p. 53.

Aneutalus S. Kèler. Menoponida. Espèce type : A. importunus (Nitzsch). Ztsch. f. Parasitenk., XI, 1939, p. 56.

Scalarisoma S. Kéler. Menovonida. Espèce type : S. heterosoma (Piaget). Zlsch.f. Purasilenk., XI, 1939, p. 57.

Colpocarenum S. Kéler. Menoponidæ. Espèce type : $C$. zonalum (Piaget). (= Menopon zonalum Piaget). Zlsch. f. Parasilenk., XI, 1939, p. 57.

\section{LANGERON.}

\section{Aphaniptères}

M sringis hubbardi G. M. Kohls. Sur Sylvilagus, Dipodomys, Peromyscus, etc (Mamm.). Etals-Unis, Montagnes Rocheuses. Publ. Heallh Rep., LIII, 19:8 p. 1217.

Meringis dipodomys G. M. Kohls. Sur Dipodomys sp. (Mamm.). Californie. Public. Heallh Rep., LIII, 1938, p. 1219. 
Rhopalopsyllus crypturi S. Wagner. Malacopsyllidæ. Cryplurus obsoletus Sta Catharinà. Sud du Brésil. Zlsch. f. Parasitenk., XI, 1939, p. 61.

Rhopalapsyllus dendrobius J. Wagner. Malacopsyllidæ. Opislhocomis hoazin. Santa Cruz, Bolivie. Zlsch. t. Parasilenk., XI, 1939, p. 63.

Neoceratophyllus Lin. Ceralophyllida. Espèce type: N. trispinosus Lin. Philippine Jl. Sc., LXX, 1939, p. 42.

Neoceratophyllus trispinosus Lin. Ceralophyllidær. Sciurus sp. (Mamm.). (.hine, Chekiang, Tien Mo Shan. Philippine Jl. Sc., LXX, 1939, p. 43. Chaetopsylla hangchowensis Lin. Vermipsyllida. Putorius sinensis (Mamm.). Chine, Hangchow. Philippine Jl. sc., LXX, 1939, p. 94.

Ctenophthalmus gil-colladol J. Wagner. Talpa sp. (Mamm.). Espagne, Madrid. Cadalso de los Vidrias. Ztsch. f. Parasilenkunde., XI, 1939, p. 236. Xenopsylla bantorum Jordan. Aethomys (Mamm.). Uganda. Novit. zool., XLI, 1938, p. 112. (1910).

Ceratophylius (Nosopsyllus) medus Jordan. Mus rallus norvegicus (Mam.). Iraq, Bagdad. Novit. zool., XL!, 1938, p. 112 (1940).

- Opisocrostis oregonensis N. E. Good. Cilellus oregonus (Merriam) (Mamm.). U.S.A., Oregon, Baker County. Publ. Health Rep., LIV, 1939, p. 1687.

Opisocrostis washingtonensis N. E. Good. Cilellus washingloni Howell (= C. townsendi auct.) (Mamm.). U.S.A. Washington, Adams County. Publ. Heallh Rep., LIV, 1939, p. 1691.

\section{LANGERON.}

\section{Hémiptères}

Eutriatoma oliveirai Neiva, Pinto et Len'. Reduvida. Brésil, Etal de Rio Grande do Sul, Porto Alegre. Mem. Inst. O. Cruz, XXXIV, 1939, p. 610.

Aradomorpha chinai A. da Costa Lima. Reduvidæ. Brésil, Matto Grosso, Maracaju. Annaes Acad. brasil. sc., XII, 1940, p. 60.

Mepraia.S. Mazza, Tobar et Jörg. Reduvidze. Espèce type : M. spinolai (Porter 1933) (= Triatoma spinolai Porter 1933). Mision de estudios de palologia regional argentina (Jujuy). Publ. n 44, Buenos Aires, 1940.

Callotriatoma Usinger. Reduvida. Espèce type : C. cubana Usinger. Univ. California publ. entomol., VII, 1939, p. 36.

Callotriatoma cubana Usinger. Reduvidx. Cuba, Loma del Gato, Cobre Range, Oriente. Univ. California publ. enlomol., VII, 1939, p. 37.

Mestor humeralis Usinger. Reduvidæ. Panama, Barro Colorado, Canal zone. Univ. California publ. entomol., VII, 1939, p. 38.

Dipetalogaster Usinger. Reduvidæ. Espèce type : D. maximus (Ulher 1894) (= Conorhinus maximus Uhler 1894). Univ. California publ. ent mol., VII, 1939, p. 40.

Triatoma nitida Usinger. Reduvidx. Guatemala, Sabranetas, La Roja. Univ. California publ. entomol., VII, 1939, p. 43.

Triatoma barberi Usinger. Reduvidæ. Mexique, Cuernavaca. Univ. California publ. entomol., VII, 1939, p. 44.

Triatoma incrassata Usinger. Rednvida. Vallée de Mexico. Univ. California publ. entomol.. V'II, 1939, p. 45.

M. LANGERON. 